OPEN ACCESS

Edited by:

Ying Zhang,

Johns Hopkins University,

United States

Reviewed by:

Tara J. Moriarty,

University of Toronto, Canada Monica E. Embers,

Tulane University, United States

${ }^{*}$ Correspondence:

Daniel P. Dulebohn

dulebohnd@niaid.nih.gov

Specialty section:

This article was submitted to

Infectious Diseases,

a section of the journal

Frontiers in Microbiology

Received: 22 May 2017

Accepted: 25 August 2017 Published: 29 September 2017

Citation:

Dulebohn DP, Richards CL, Su H, Lawrence KA and Gherardini FC (2017) Weak Organic Acids Decrease Borrelia burgdorferi Cytoplasmic $\mathrm{pH}$, Eliciting an Acid Stress Response and Impacting RpoN-

and RpoS-Dependent Gene

Expression. Front. Microbiol. 8:1734. doi: 10.3389/fmicb.2017.01734

\section{Weak Organic Acids Decrease Borrelia burgdorferi Cytoplasmic pH, Eliciting an Acid Stress Response and Impacting RpoN- and RpoS-Dependent Gene Expression}

\author{
Daniel P. Dulebohn*, Crystal L. Richards, Hua Su, Kevin A. Lawrence and \\ Frank C. Gherardini
}

Laboratory of Zoonotic Pathogens, Gene Regulation Section, Division of Intramural Research, Rocky Mountain Laboratories, National Institute of Allergy and Infectious Diseases, National Institutes of Health, Hamilton, MT, United States

The spirochete Borrelia burgdorferi survives in its tick vector, Ixodes scapularis, or within various hosts. To transition between and survive in these distinct niches, B. burgdorferi changes its gene expression in response to environmental cues, both biochemical and physiological. Exposure of $B$. burgdorferi to weak monocarboxylic organic acids, including those detected in the blood meal of fed ticks, decreased the cytoplasmic $\mathrm{pH}$ of $B$. burgdorferi in vitro. A decrease in the cytoplasmic $\mathrm{pH}$ induced the expression of genes encoding enzymes that have been shown to restore $\mathrm{pH}$ homeostasis in other bacteria. These include putative coupled proton/cation exchangers, a putative $\mathrm{Na}^{+} / \mathrm{H}^{+}$antiporter, a neutralizing buffer transporter, an amino acid deaminase and a proton exporting vacuolar-type $\mathrm{V}_{0} \mathrm{~V}_{1}$ ATPase. Data presented in this report suggested that the acid stress response triggered the expression of RpoN- and RpoS-dependent genes including important virulence factors such as outer surface protein $\mathrm{C}(\mathrm{OspC})$, BBA66, and some BosR (ㅌorrelia oxidative stress regulator)-dependent genes. Because the expression of virulence factors, like OspC, are so tightly connected by RpoS to general cellular stress responses and cell physiology, it is difficult to separate transmission-promoting conditions in what is clearly a multifactorial and complex regulatory web.

Keywords: Lyme disease, gene regulation, arthropod vector, acid stress response, virulence factor expression

\section{INTRODUCTION}

Pathogens disseminated by arthropod vectors are responsible for many emerging and reemerging infectious diseases that impose a significant burden on public health worldwide. Successful vectorborne transmission requires the pathogen to survive and replicate in very dissimilar hosts. Typically, bacterial pathogens sense, respond, and adapt to host environments during vectorhost-vector (infectious cycle) transitions. These adaptations ensure that the appropriate changes in gene expression occur at the time suitable for successful colonization of these habitable hosts. 
Identifying the environmental and biochemical changes that occur in these distinct niches and understanding how pathogens respond during the infectious cycle can provide valuable insight into the pathogenesis of vector-borne pathogens.

The hard tick Ixodes scapularis can transmit pathogenic protozoans, viruses, and bacteria, including the causative agent of Lyme disease, the spirochete, Borrelia burgdorferi (Burgdorfer et al., 1982; Steere et al., 1983; Nelder et al., 2016). B. burgdorferi survives strictly in a vector- or host-environment (Lane et al., 1991) and successfully transitions between these to complete its infectious cycle. Transmission from ticks and establishment of infection in a host requires an atypical signaling cascade, the Rrp2-RpoN-RpoS cascade, to direct key changes in gene expression (Yang et al., 2003; Fisher et al., 2005; Dunham-Ems et al., 2012). This signaling cascade consists of the cytoplasmic response regulator protein-2 (Rrp2) and two alternative sigma factors, RpoN and RpoS. Activation of Rrp 2 promotes RpoNdirected transcription of rpoS and subsequent RpoS-directed transcription of genes encoding proteins required for survival and key virulence factors (Hübner et al., 2001; Clifton et al., 2006; Burtnick et al., 2007; Caimano et al., 2007; Samuels, 2011).

Multiple environmental parameters fluctuate during the infectious cycle and changes in $\mathrm{pH}(7.5 \rightarrow 6.8)$, temperature $\left(25^{\circ} \rightarrow 34^{\circ}\right)$, osmolarity $(600 \mathrm{mOsm} \rightarrow 300 \mathrm{mOsm})$, nutrient availability and cell density $\left(\sim 1-2 \times 10^{8}\right.$ cells $\left./ \mathrm{mL}\right)$ have been shown to trigger the expression of RpoS-dependent virulence factors in vitro (Schwan et al., 1995; Carroll et al., 1999; Yang et al., 2000; Bontemps-Gallo et al., 2016). Additionally, cells encounter reactive nitrogen species (RNS) and reactive oxygen species (ROS) during the infectious cycle (Bourret et al., 2016). These stressors can promote changes in gene expression (Hyde et al., 2006) and require protective stress response proteins (e.g., UvrB, MutS; Bourret et al., 2016; Ramsey et al., 2017), some directly regulated by the Borrelia oxidative stress regulator, BosR (e.g., SOD, CoADR), for survival (Boylan et al., 2006; EsteveGassent et al., 2009; Eggers et al., 2011). Moreover, acetate (Xu et al., 2010; Van Laar et al., 2012; Richards et al., 2015) and benzoate (Richards et al., 2015) activate RpoS expression, utilizing a mechanism that is independent of the acetate-derived acetyl-phosphate (Ac-P) phosphorylation of Rrp2 (Richards et al., 2015). How weak monocarboxylic acids affect the physiology and gene expression in B. burgdorferi is unclear.

Membrane-permeable weak monocarboxylic organic acids, like acetate and benzoate, have been shown to trigger acid/proton stress in some bacteria (Kirkpatrick et al., 2001). This stress occurs when excess, free protons accumulate in the cytoplasm (Foster, 1999). Protonated weak acids can diffuse across the cell membrane, dissociate in the cytoplasm, and increase the concentration of intracellular protons. This generates differences in the $\mathrm{pH}$ between the external environment $\left(\mathrm{pH}_{\mathrm{e}}\right)$ and the cytoplasm $\left(\mathrm{pH}_{\mathrm{i}}\right)$, acidifies the cytosol, decreasing the activity of $\mathrm{pH}$-sensitive enzymes, and disrupting the proton motive force (PMF) (Bearson et al., 1997; Foster, 1999, 2004; Kanjee and Houry, 2013). To survive, many bacteria initiate an acid stress response to restore $\mathrm{pH}$ homeostasis. Disparate mechanisms are employed to reestablish a favorable $\mathrm{pH}_{i}$ including: (i) coupled proton exchange with cations or anions (antiporters); (ii) generating or transporting neutralizing buffering molecules including proteins (ionizable side groups can act as cytosolic buffers), polyamines, polyphosphates, and inorganic phosphates; (iii) utilizing amino acid decarboxylases and/or deaminases; (iv) the synthesis of acid resistant fatty acids and lipids; and (v) reversing proton import via the $\mathrm{F}_{1} / \mathrm{F}_{\mathrm{o}}$ ATPase by hydrolyzing ATP to exclude protons (Bearson et al., 1997; Foster, 1999; Slonczewski et al., 2009). Many of the systems utilized by other bacteria to neutralize excess protons in the cytoplasm are absent in B. burgdorferi, including amino acid synthesis, amino acid decarboxylases, fatty acid biosynthesis, most neutralizing metabolites, an $\mathrm{F}_{1} / \mathrm{F}_{\mathrm{o}}$ ATPase and some proton pumping transporters (Fraser et al., 1997). Lacking a respiratory chain or cytochrome system for eliminating protons from the cytoplasm, $B$. burgdorferi utilizes a proton pumping $\mathrm{V}_{\mathrm{o}} \mathrm{V}_{1}$ vacuolar-type ATPase that hydrolyzes ATP and pumps protons to maintain the essential PMF (Fraser et al., 1997; Radolf and Samuels, 2010). The paucity of recognizable mechanisms to deal with perturbations of cytoplasmic $\mathrm{pH}$ suggests that $B$. burgdorferi is not exposed to significant levels of acids $(\mathrm{HCl})$ or weak monocarboxylic organic acids or is using an atypical mechanism(s) to deal with acidification of its cytosol. However, the molecular mechanism and physiological significance of the acetate/benzoate stimulated changes in gene expression was previously unclear (Xu et al., 2010; Richards et al., 2015).

To understand the molecular mechanism and biological significance of the acetate-induced changes in gene expression and determine if this induction is related to a typical acid stress response, we analyzed the effect of membrane-permeable acids on B. burgdorferi. We demonstrated that membrane-permeable acids decrease $\mathrm{pH}_{\mathrm{i}}$ and exposure to the membrane permeable acid propionate activates a previously uncharacterized acid stress response. Exposure to monocarboxylic organic acids triggered an increase in the transcription of genes encoding proteins that represent a unique approach to relieving acid stress. Moreover, our data established that membrane-permeable acids are present during the infectious cycle in both mouse and rabbit sera, and in tick midgut contents following a blood meal. These monocarboxylic acids may act as environmental cues during the infectious cycle to trigger changes in gene expression to generate protective responses to multiple stressors and likely promote long-term survival of $B$. burgdorferi throughout the infectious cycle.

\section{RESULTS}

\section{Membrane-Permeable Acids Decrease Cytoplasmic pH in B. burgdorferi}

The addition of acetate to $B$. burgdorferi growth medium promotes the expression of RpoS and RpoS-dependent genes (e.g., OspC, DbpA, etc.; Xu et al., 2010; Van Laar et al., 2012; Richards et al., 2015). The acetate-triggered induction was presumed to be a result of increased levels of intracellular Ac-P, which, in turn, initiated the Rrp2-RpoN-RpoS regulator cascade (Xu et al., 2010). However, a recent study has shown that acetate can upregulate RpoN/RpoS-dependent proteins in the absence 
of Ac-P (Richards et al., 2015). The molecular mechanism and physiological significance of the acetate-triggered induction of virulence factors has remained unclear.

One consequence of exposing bacterial cells to membrane permeable acids, like acetate, is a drop in $\mathrm{pH}_{\mathrm{i}}$ (acid stress) which can retard growth and, under extreme conditions, cause cell death (Foster, 1999; Wilks and Slonczewski, 2007; Slonczewski et al., 2009; Wilks et al., 2009). Since acetate-induced expression of OspC and RpoS was independent of Ac-P concentration, it seemed likely that this "acetate effect" might result from acetate directly decreasing $\mathrm{pH}_{\mathrm{i}}$. To test this possibility, we monitored the cytoplasmic $\mathrm{pH}$ of $B$. burgdorferi exposed to various membrane-permeable acids using the $\mathrm{pH}$-responsive membrane-permeable dye pHrodo Green (effective $\mathrm{pH}$ range 4-7) (Miksa et al., 2009). B. burgdorferi B31 strain A3 spirochetes were grown in BSK-II media containing acetate, benzoate, lactate, or propionate, stained with pHrodo Green and visualized using fluorescence microscopy (Figure 1A). Spirochetes grown in BSK-II alone and exposed to pHrodo Green showed little fluorescence compared to background indicating a near neutral intracellular $\mathrm{pH}$ (Figure 1A, untreated). However, spirochetes grown in media containing acetate, benzoate, or propionate and exposed to the dye were demonstrably brighter, suggesting that these acids had acidified the cytosol. Cells exposed to lactate showed little change in fluorescence, indicating that this acid had a minor effect on intracellular $\mathrm{pH}$ (Figure 1A, lactate). We confirmed the fluorescence microscopy data using a more sensitive quantitative fluorescence microplate assay as described in "Materials and Methods" (Figure 1B). Cells exposed to acetate, benzoate, or propionate had a statistically significant increase in fluorescence compared to the untreated control (Figure 1B, untreated, acetate, benzoate, propionate) while lactate (Figure 1B, lactate) showed no change in fluorescence relative to the control. Although fluorescence microscopy showed a slight change in fluorescence for cells exposed to lactate, there was no change in the microplate assay, suggesting that subtle changes in fluorescence are difficult to quantify using this assay. Additionally, the $\mathrm{pH}$ of the buffered media did not change throughout the experiment. The result for lactate was predictable as B. burgdorferi is a homofermenter whose sole endproduct of central metabolism is lactate. Thus, these bacteria have a highly efficient lactate secretion system that would actively excrete lactate as fast as it could enter the cell. These data suggest that membrane permeable acids can directly affect the $\mathrm{pH}_{\mathrm{i}}$ of B. burgdorferi.

\section{A Decrease in $\mathrm{pH}_{\mathrm{i}}$ Promotes $\mathrm{RpoN}$ - and RpoS-Dependent Virulence Factor Expression}

Having demonstrated that membrane-permeable weak acids decrease $\mathrm{pH}_{\mathrm{i}}$, we next sought to determine if the induction of RpoS and OspC in the presence of acetate ( $\mathrm{Xu}$ et al., 2010; Van Laar et al., 2012; Richards et al., 2015) is due to an acid stress response in B. burgdorferi. We analyzed the effect of acetate, benzoate, lactate, and propionate on RpoS and RpoS-dependent virulence factors OspC and BBA66 during mid-logarithmic phase $\left(1-5 \times 10^{7}\right.$ spirochetes $\left./ \mathrm{mL}\right)$ to avoid growth-dependent ("stationary phase") induction of RpoS (Yang et al., 2000). Cell lysates of spirochetes grown in BSK-II containing acetate, benzoate, lactate, or propionate were analyzed by immunoblot for production of RpoS, OspC, and BBA66. Benzoate and propionate had the greatest effect on RpoS expression, causing a marked increase in RpoS expression compared to the untreated control (Figure 1C). Acetate led to a moderate increase in RpoS levels while lactate did not increase RpoS expression. Benzoate and propionate, followed by acetate, resulted in the greatest increase in expression of the RpoS-dependent factors OspC and BBA66 (Figure 1C and Supplementary Figure S1). Lactate increased the expression of only OspC compared to the untreated control (Figure 1C and Supplementary Figure S1). Taken together, these data suggest that a decrease in $\mathrm{pH}_{\mathrm{i}}$ promotes the expression of RpoS and RpoS-dependent virulence factors, with non-metabolizable acids having the greatest affect on both RpoS production and $\mathrm{pH}_{\mathrm{i}}$.

Synthesis of RpoS and RpoS-dependent virulence factors requires activation of the Rrp2-RpoN-RpoS signal transduction cascade (Yang et al., 2003; Fisher et al., 2005; Burtnick et al., 2007) and the transcriptional regulator BosR (Ouyang et al., 2009). To determine if acid stress promotes production of OspC and BBA66 through the Rrp2-RpoN-RpoS signaling pathway, B. burgdorferi strains B31-A3 (wild-type, WT), B31-A3 $\Delta r p o N$ $(\Delta r p o N), \mathrm{B} 31-\mathrm{A} 3 \Delta r p o S(\Delta r p o S)$, and B31-A3 $\Delta$ bosR $(\Delta b o s R)$ were grown in medium containing benzoate and we determined the level of OspC and BBA66 by immunoblot (Figure 1D). Unlike the WT strain, in the $\triangle r p o N, \triangle r p o S$, and $\triangle b o s R$ mutant strains, production of OspC and BBA66 were abrogated (Figure 1D). Therefore, in response to membrane permeable acids, synthesis of RpoS and RpoS-dependent virulence factors likely proceed through the Rrp2-RpoN-RpoS pathway and this response requires BosR. These results suggest a generalized response to membrane-permeable acids where a decrease in $\mathrm{pH}_{\mathrm{i}}$ activates the Rrp2-RpoN-RpoS/BosR signaling cascade resulting in virulence factor synthesis in B. burgdorferi.

\section{Membrane-Permeable Acids Activate the Rrp2-RpoN-RpoS Signaling Cascade and the Oxidative Stress Regulon}

Regulation of the Rrp2-RpoN-RpoS cascade in B. burgdorferi is complex, with multiple components regulated transcriptionally, post-transcriptionally, or post-translationally (Yang et al., 2003; Fisher et al., 2005; Burtnick et al., 2007; Lybecker and Samuels, 2007; Dulebohn et al., 2014; Richards et al., 2015). Having established that RpoS increases in response to acid stress, we next sought to determine if rpos, or other key components of the Rrp2-RpoN-RpoS cascade, are transcriptionally regulated in response to membrane permeable acids. Using quantitative reverse transcriptase PCR (qRT-PCR) we analyzed the level of $\operatorname{rrp} 2$ and $r p o N$ in response to membrane permeable acids and found they were relatively unchanged under acid stress conditions (Figure 2A). Conversely, rpoS transcript levels demonstrated a statistically significant increase under the 


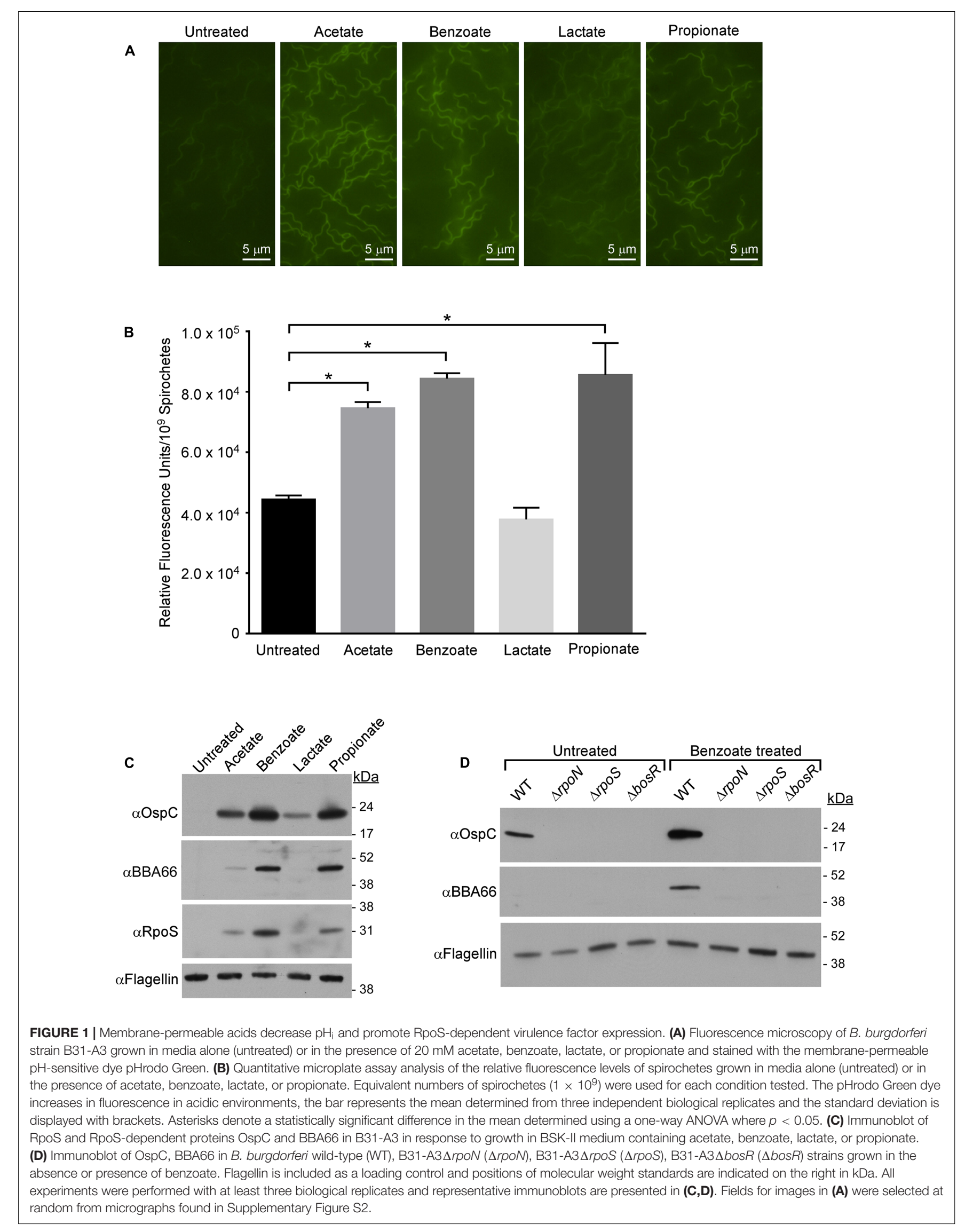



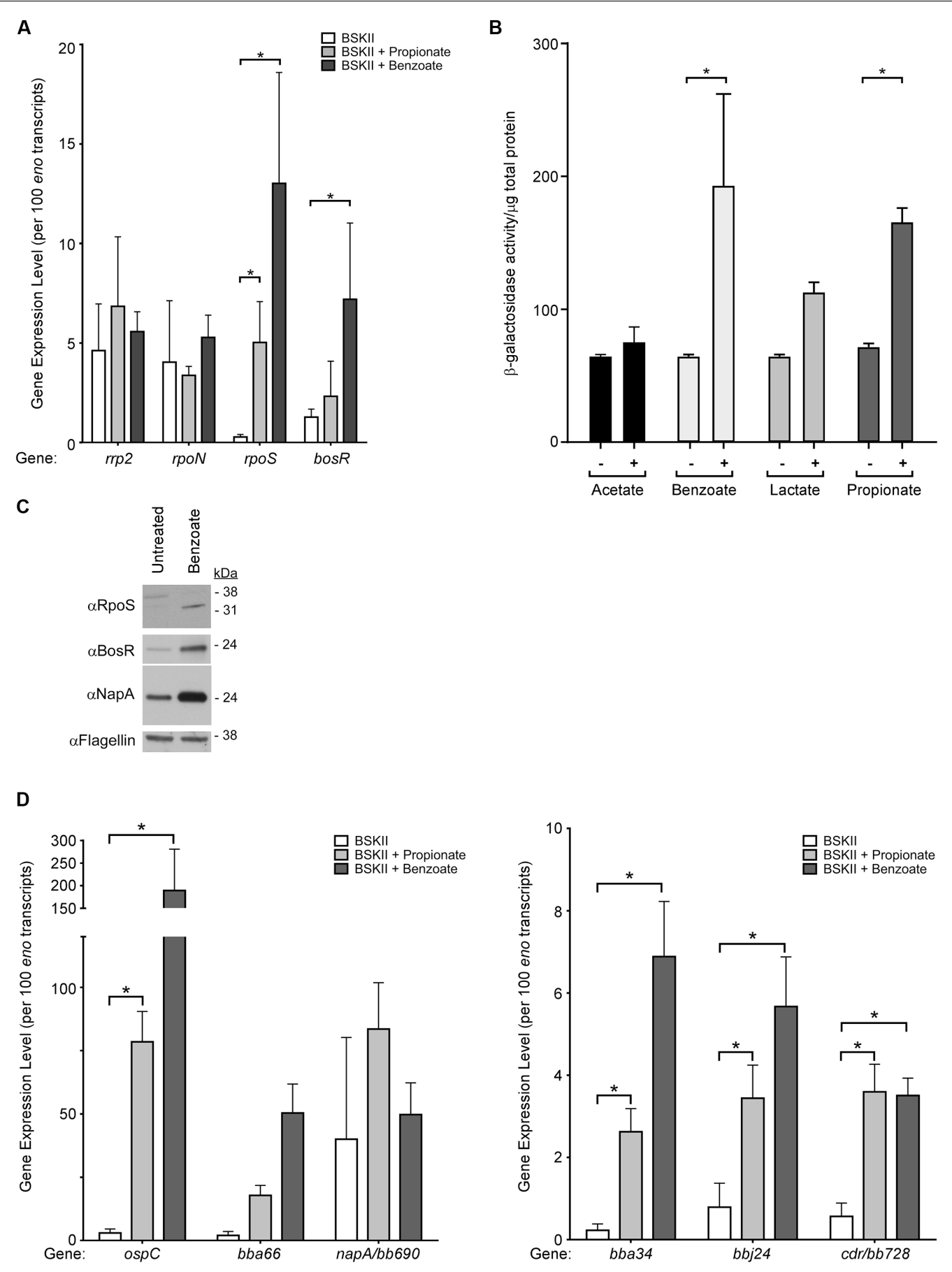

FIGURE 2 | Membrane-permeable acids activate transcription of RpoS-dependent virulence factors and the oxidative stress regulon. Gene expression and immunoblot analysis of $B$. burgdorferi strain B31-A3 in response to membrane-permeable acids. (A) qRT-PCR analysis of key regulators of the Rrp2-RpoN-RpoS/BosR signal transduction cascade, rrp2, rpoN, rpoS, and bosR. (B) B. burgdorferi strain B31-A3/pBSV2G-rpoSp-lacZ $\mathrm{Bb}$ containing a shuttle vector with the rpoS promoter fused to $\mathrm{lac}_{\mathrm{Bb}}$ was grown in the $(-)$ absence or $(+)$ presence of acetate, benzoate, lactate, or propionate and $\beta$-galactosidase activity in cell lysates was analyzed. Brackets with asterisks indicate a statistically significant difference between the means with $p$-values $<0.05$ determined using a two-way ANOVA. (C) Immunoblot analysis of RpoS, BosR, and the BosR-regulated factor NapA/BB690 in B. burgdorferi in response to benzoate. Flagellin is included as a loading control and the position of molecular weight standards is indicated on the right in kDa. (D) qRT-PCR analysis of RpoS-dependent genes ospC, bba66, bba34, bbj24 and BosR-regulated genes napA/bb690 and cdr/bb728. Expression levels were normalized to the eno gene encoding enolase and are represented as transcript copies per 100 eno copies. Asterisks denote a statistically significant difference of the mean determined using a two-way ANOVA with $p<0.05$. All experiments were performed using at least three biological replicates with the mean and standard deviation displayed in (A,B,D) and a representative immunoblot presented in (C). 
same conditions, suggestive of transcriptional regulation of rpoS under acid stress and consistent with the immunoblot data demonstrating an increase in RpoS (Figures 1C, 2C). Additionally, $B$. burgdorferi strain B31-A3 harboring a plasmid containing the rpoS promoter fused to the $l a c Z_{\mathrm{Bb}}$ gene (Hayes et al., 2010) exhibited a significant increase in $\beta$-galactosidase activity in the presence of benzoate or propionate (Figure 2B).

The level of the rpoS transcript expressed in B. burgdorferi is intricately linked to the BosR (Ouyang et al., 2009) and BosR is required for expression of $\mathrm{OspC}$ and $\mathrm{BBA} 66$ in response to acid stress (Figure 1D). We quantified the level of bosR transcript and found that expression remained relatively unchanged in the presence of propionate, but increased significantly in spirochetes exposed to benzoate (Figure 2A). Having observed a statistically significant increase in bos $R$ transcript levels in response to benzoate we further evaluated BosR and the BosR-regulated protein NapA/BB0690 by immunoblot. In response to benzoate, both BosR and NapA levels increased compared to the untreated control (Figure 2C). Taken together, these data suggest that rpoS and bosR transcript levels increase in response to acid stress, leading to increased levels of these key regulators that may affect expression of both RpoS-dependent and BosR-regulated genes, suggestive of a possible overlap between an acid stress response and the oxidative stress response in B. burgdorferi.

Since RpoS and BosR levels increased under acid stress, we quantified RpoS-dependent and BosR-regulated transcripts in response to acid stress by qRT-PCR. These included the RpoS-dependent transcripts ospC, bba66, bbj24, bba34 and the BosR-regulated genes napA/bb690 and $c d r / b b 728$ (Figure 2D). B. burgdorferi was grown in BSK-II supplemented with benzoate or propionate and transcript levels indicated that the RpoSdependent genes ospC, bba66, bbj24, and bba34, were induced in response to propionate or benzoate compared to the untreated control (Figure 2D). Consistent with these data, $\beta$-galactosidase activity was significantly increased in spirochetes containing a plasmid with the $l a c Z_{\mathrm{Bb}}$ gene fused to the ospC promoter in the presence of acetate, benzoate, or propionate (Supplementary Figure S3). Transcript levels of the BosRregulated gene (Boylan et al., 2003; Hyde et al., 2006, 2009; Ouyang et al., 2009) napA/bb690 remained relatively unchanged, however, the level of $c d r / b b 728$, encoding CoA reductase, was significantly increased in response to both propionate and benzoate (Figure 2D). These data indicate that RpoSdependent and a BosR-dependent genes are induced in response to acid stress, also suggestive of an overlap between an acid stress response and the oxidative stress response in B. burgdorferi.

\section{Propionate Promotes Transcription of Genes Typically Associated with Protection from Acid Stress}

In response to acid stress, many bacteria activate an acid stress response (Marquis et al., 1987; Bearson et al., 1997; Foster, 1999, 2004; Slonczewski et al., 2009; Kanjee and Houry, 2013) to stabilize $\mathrm{pH}_{\mathrm{i}}$ and retain the PMF (Bearson et al., 1997;
Slonczewski et al., 2009; Wilks et al., 2009). B. burgdorferi is thought to lack "typical" bacterial stress responses (Caimano et al., 2004) and is missing many of the enzymes and transporters characteristically utilized to mitigate acid stress (Fraser et al., 1997). However, the genome does encode a vacuolar-type $V_{0} V_{1}$ ATPase, an arginine deiminase system (ArcA-ArcB-ArcD), and several proton/ion pumping transporters/symporters that could play a role in maintaining $\mathrm{pH}_{\mathrm{i}}$ and the $\mathrm{PMF}$ in response to acid stress. To determine if $B$. burgdorferi responds to acid stress by increasing the transcription of genes encoding protective enzymes or transporters associated with an acid stress response we monitored select genes in response to spirochete growth under acid stress conditions. To generate acid stress conditions, we exposed spirochetes to propionate, as it activates the Rrp2RpoN-RpoS cascade (Figure 1C) and unlike lactate and acetate, is not actively exported from Borrelia or utilized in undecaprenylP synthesis (Fraser et al., 1997). Unable to be metabolized like acetate or exported like lactate, propionate may accumulate in the cytoplasm, enhancing its ability to decrease $\mathrm{pH}_{\mathrm{i}}$. Additionally, the tick microbiome does not harbor bacteria predicted to generate benzoate, however, several species of bacteria found in the I. scapularis midgut could potentially produce propionate as a metabolic end-product, likely exposing $B$. burgdorferi to this acid during the infectious cycle (Narasimhan et al., 2014).

The Borrelia $\mathrm{V}_{\mathrm{o}} \mathrm{V}_{1}$ ATPase (Fraser et al., 1997) is hypothesized to hydrolyze ATP to translocate protons out of the cytoplasm to maintain the PMF and drive proton-coupled transport (Radolf and Samuels, 2010). This is uncommon in bacteria but vacuolar $\mathrm{V}_{\mathrm{o}} \mathrm{V}_{1}$ ATPases are often utilized by eukaryotic cells to acidify intracellular compartments through the ATPdriven translocation of protons (Nishi and Forgac, 2002; Mindell, 2012). To determine if the $\mathrm{V}_{\mathrm{o}} \mathrm{V}_{1}$ ATPase was playing a role in the acid stress response we quantified the transcript levels of the $\mathrm{V}_{\mathrm{o}} \mathrm{V}_{1}$ ATPase components (bb090, bb091, bb092, $b b 093, b b 094, b b 096)$. We found that all six components of the $\mathrm{V}_{0} \mathrm{~V}_{1}$ ATPase had increased expression in response to propionate (Figure 3A). The most significant increases were in the $\mathrm{V}_{1}$ ATP-binding components $($ bb093, bb096) and the $\mathrm{V}_{\mathrm{o}}$ proton translocation (bb091) component of the $\mathrm{V}_{\mathrm{o}} \mathrm{V}_{1}$ ATPase. We also quantified the expression of the arginine deiminase pathway genes ( $\operatorname{arcA} / b b 841-\operatorname{arcB} / b b 842-a r c D / b b 843)$, whose products can protect bacteria from acid stress through the ArcA-mediated conversion of arginine to citrulline and ammonia (Marquis et al., 1987; Ryan et al., 2009; Cusumano and Caparon, 2015). All of these genes had increased expression levels in response to propionate with statistically significant increases in both $a r c B / b b 842$ and $\operatorname{arcD} / b b 843$ transcript levels (Figure 3A).

In response to acid stress, bacteria must maintain both the $\mathrm{pH}_{\mathrm{i}}$ and their PMF to survive (Bearson et al., 1997; Foster, 1999, 2004; Slonczewski et al., 2009). B. burgdorferi encodes several transporters/symporters that could play important roles in maintaining PMF homeostasis under acid stress conditions through the transport of protons/ions or monocarboxylic acids. We found that specific transport systems were induced in response to acid stress. Specifically, a Na/H $\mathrm{H}^{+}$transporter (bb638), glutamic acid transporter (bb729), potassium transporters 


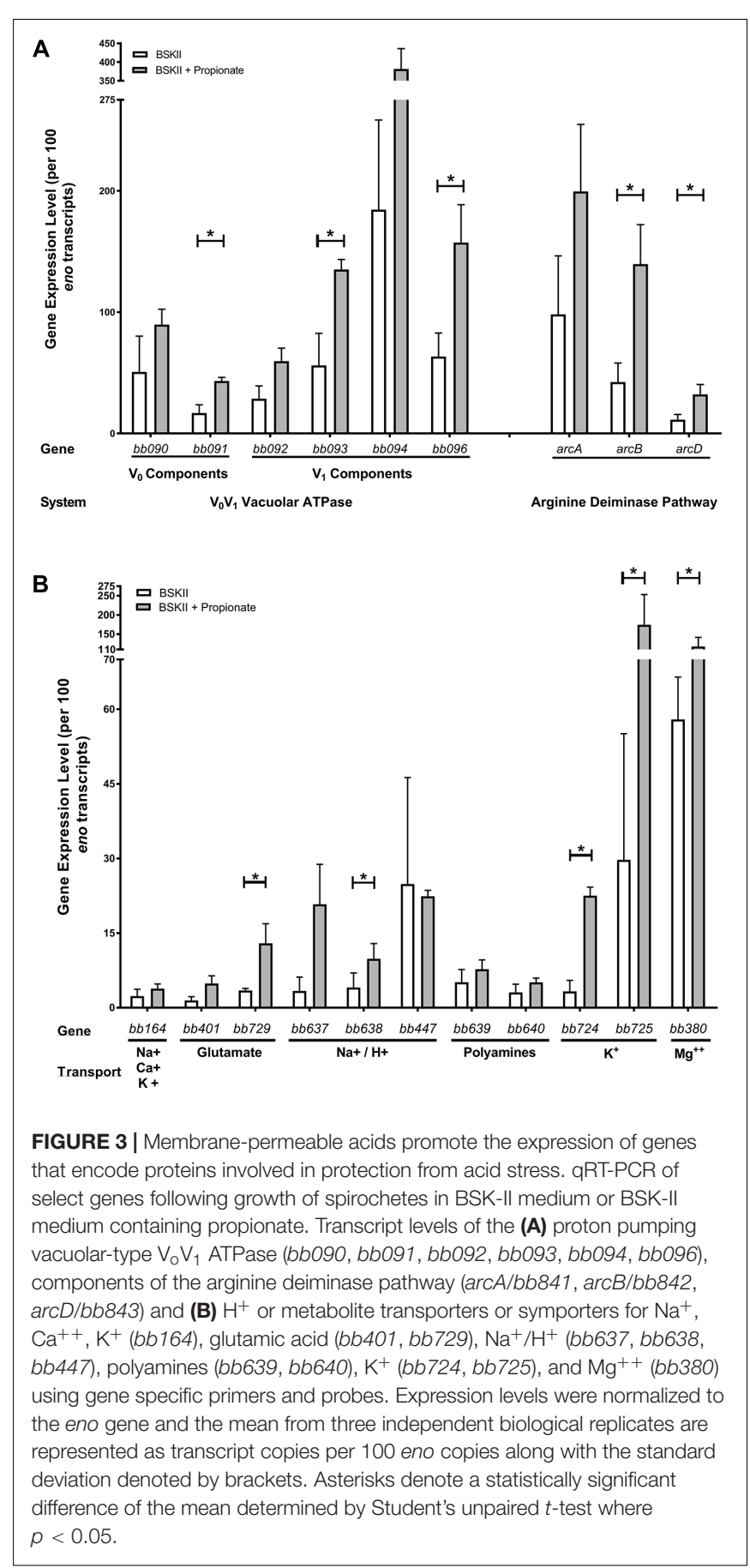

(bb724, bb725), and a magnesium transporter (bb380) were all significantly induced in response to propionate (Figure $3 \mathbf{B}$ ). However, the $\mathrm{Na}^{+}, \mathrm{Ca}^{++}, \mathrm{K}^{+}$transporter bb164 and the $\mathrm{Na}^{+} / \mathrm{H}^{+}$ transporter $b b 447$ remained relatively unchanged (Figure 3B). Taken together, these results suggest that in response to acid stress, B. burgdorferi initiates an acid stress response that promotes the transcription of select genes encoding proteins that can potentially mitigate the detrimental effects of excess protons in the cytoplasm.

\section{Arginine Inhibits Acidification of the B. burgdorferi Cytoplasm}

We next sought to define conditions that would inhibit acidification of the cytoplasm or inhibit permeable acidmediated activation of the Rrp2-RpoN-RpoS pathway. To test if arginine could prevent the acidification of the B. burgdorferi cytoplasm, either through direct, or indirect buffering through the deamination of arginine and production of ammonia, we again monitored $\mathrm{pH}_{\mathrm{i}}$ using the $\mathrm{pH}$-sensitive fluorescent dye pHrodo Green. B. burgdorferi was grown in media containing benzoate, benzoate and arginine, or benzoate and alanine, stained with pHrodo Green and intracellular fluorescence was evaluated by fluorescence microscopy (Figure 4A). Benzoate has been used to study the acid stress response in $E$. coli due to its ability to decrease $\mathrm{pH}_{\mathrm{i}}$ and induce a robust acid stress response (Wilks and Slonczewski, 2007; Slonczewski et al., 2009), and in B. burgdorferi benzoate had the greatest effect on $\mathrm{pH}_{\mathrm{i}}$ and $\mathrm{OspC}$ and $\mathrm{BBA} 66$ expression. Therefore, we utilized benzoate to promote acid stress and to make any reduction in fluorescence or protein expression easily detectable in the presence of arginine. Spirochetes grown in BSK-II alone showed weak fluorescence, indicating a near neutral $\mathrm{pH}$ (Figure 4A, untreated) and spirochetes grown in BSK-II containing benzoate were markedly brighter, suggesting a decreased $\mathrm{pH}_{\mathrm{i}}$ (Figure $\mathbf{4 A}$, benzoate). However, spirochetes grown in both benzoate and arginine had fluorescence levels similar to the untreated controls, suggesting a near neutral $\mathrm{pH}_{\mathrm{i}}$ (Figure 4A, benzoate + arginine). To test whether this buffering effect was specific to arginine, spirochetes were grown in BSK-II containing benzoate and alanine and fluorescence levels were found to be equivalent to the benzoate treated samples (Figure 4A, benzoate + alanine). To better characterize the effects of arginine on permeable acid-mediated changes to $\mathrm{pH}_{\mathrm{i}}$, we next measured $\mathrm{pH}_{\mathrm{i}}$ changes using pHrodo Green in a quantitative microplate assay. Similar to what we had seen by fluorescence microscopy, spirochetes grown in the presence of benzoate had significantly higher relative fluorescence levels in the microplate assay (Figure 4B). Unlike spirochetes grown in benzoate, spirochetes grown in both benzoate and arginine had relative fluorescence levels similar to the untreated controls (Figure 4B). The relative fluorescence levels of spirochetes grown in media containing benzoate and alanine were equivalent to the benzoate treated samples (Figure 4B). These results indicate that supplementing with arginine can inhibit the acidification of the B. burgdorferi cytoplasm that occurs in response to benzoate.

\section{Activation of the Rrp2-RpoN-RpoS Signaling Cascade Is Dependent on $\mathrm{pH}_{\mathrm{i}}$ and Independent of the Histidine Kinase Hk2}

If the Rrp2-RpoN-RpoS cascade is activated due to a decrease in $\mathrm{pH}_{\mathrm{i}}$, then the addition of arginine should inhibit induction of the Rrp2-RpoN-RpoS cascade. To test this hypothesis, we grew $B$. burgdorferi in the presence of both benzoate and arginine and monitored OspC and BBA66 levels by immunoblot. To ensure that any affect attributed to arginine was specific to arginine, WT 

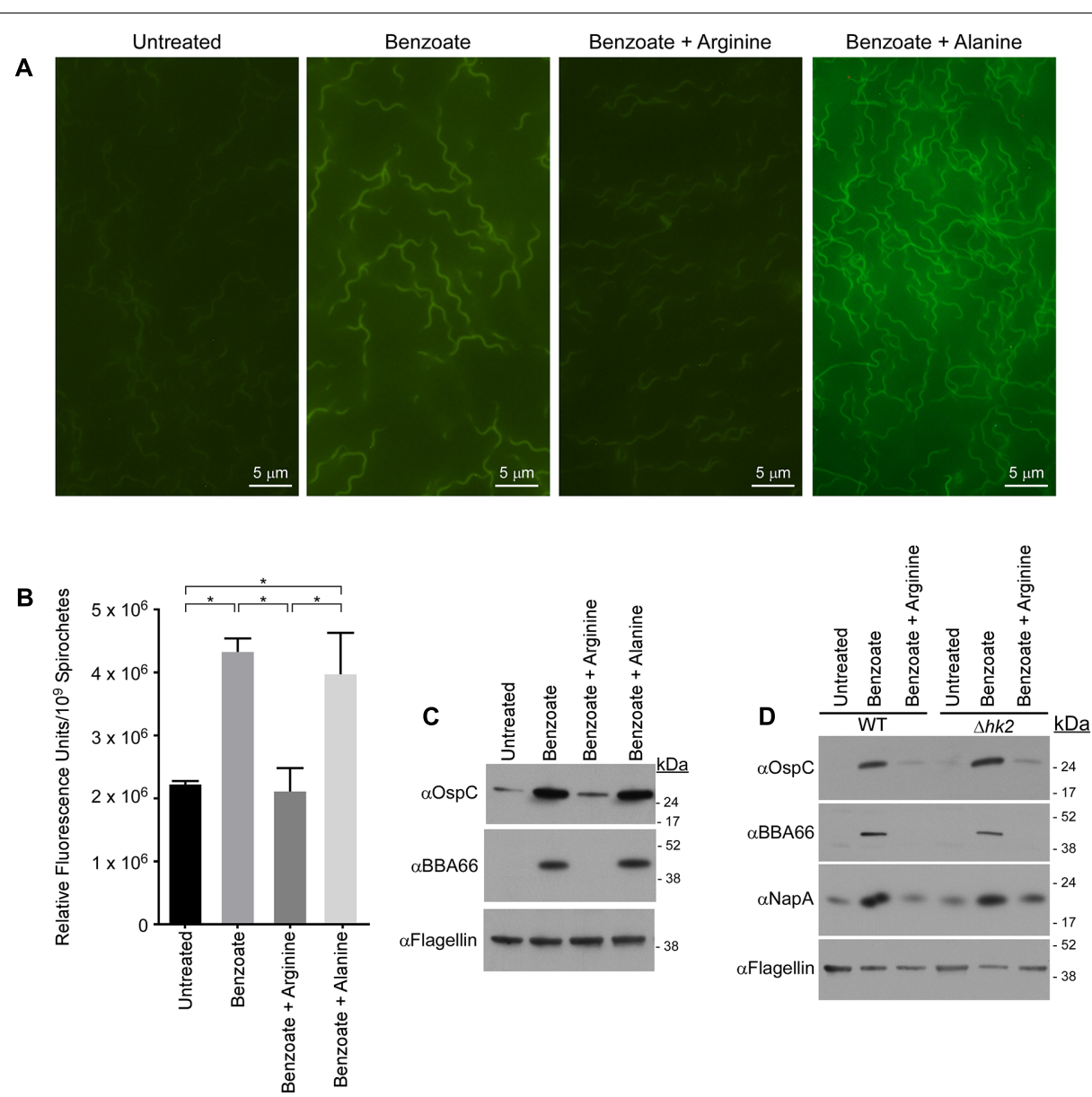

FIGURE 4 | Arginine inhibits acidification of the B. burgdorferi cytoplasm and prevents induction of RpoS-regulated proteins independent of the histidine kinase Hk2. (A) Fluorescence microscopy or (B) quantitative microplate assay of spirochetes grown in media alone (untreated), or in the presence of 20 mM benzoate, benzoate and arginine, or benzoate and alanine and stained with the membrane-permeable pH-sensitive dye pHrodo Green. Increased fluorescence indicates a decrease in $\mathrm{pH}$ and the mean value from three biological replicates is shown with the standard deviation. Asterisks denote a statistically significant difference of the mean determined using a one-way ANOVA where $p<0.05$. (C) Immunoblot of OspC and BBA66 in B. burgdorferi cells treated with benzoate, benzoate and arginine, or benzoate and alanine. (D) Immunoblot of cell lysates from B. burgdorferi B31-A3 (WT) and B. burgdorferi B3-A3 $\Delta$ hk2 ( $\Delta$ hk2) for RpoS-dependent virulence factors OspC, BBA66 and the BosR-regulated factor NapA/BB690 in response to growth in BSK-II or BSK-II supplemented with benzoate, or benzoate and arginine. Flagellin levels were used as a loading control and the position of molecular weight standards is indicated on the right in kDa. With the exception of the microplate assay which was performed in biological duplicate and technical triplicate, all experiments were performed with three biological replicates and representative immunoblots are presented in (C,D). Images for (A) were selected at random from images found in Supplementary Figure S2.

B31-A3 spirochetes were cultured in BSK-II containing benzoate and alanine. Levels of OspC and BBA66 were again increased in response to benzoate, however, not in the presence of both benzoate and arginine (Figures 4C,D). The addition of alanine was unable to inhibit the permeable acid-mediated induction of OspC and BBA66 and arginine alone did not promote activation of the Rrp2-RpoN-RpoS cascade (data not shown). Taken together with our previous data, these results suggest that $\mathrm{pH}_{\mathrm{i}}$ and virulence factor expression are coupled in $B$. burgdorferi. A decrease in $\mathrm{pH}_{\mathrm{i}}$ can activate the Rrp2-RpoN-RpoS cascade and promote virulence factor expression.

We next focused on the molecular mechanism underlying the activation of the Rrp2-RpoN-RpoS cascade under acid stress. The response regulator Rrp2 is essential in B. burgdorferi (Groshong et al., 2012) and its cognate histidine kinase-2 (Hk2) has no known function. We tested whether $\mathrm{Hk} 2$ might play a role in activation of the Rrp2-RpoN-RpoS cascade under acid stress. Strains B31-A3 (WT) and B31-A $3 \Delta h k 2(\Delta h k 2)$ B. burgdorferi (Xu et al., 2010) were grown in BSK-II media supplemented with benzoate or benzoate and arginine and immunoblots of cell lysates demonstrated that OspC and BBA66 were increased in response to benzoate, but not in the presence of benzoate and arginine (Figure 4D). Additionally, we analyzed the level of NapA/BB690 levels to determine what affect benzoate and arginine would have, if any, on the oxidative stress response. Similar to what we observed for OspC and BBA66, NapA/BB690 levels increased in the presence of benzoate and levels were similar to the untreated sample in the benzoate and arginine treated samples (Figure 4D). These data indicate that $\mathrm{Hk} 2$ does not play a role in the permeable acid-mediated induction 
of RpoS-dependent factors (Figure 4D), or in promoting the expression of NapA/BB690. These results are consistent with what has been previously shown for the acetate-triggered induction of RpoS (Xu et al., 2010) and Hk2 does not play a role in the arginine-mediated inhibition of the Rrp2-RpoN-RpoS cascade or in expression of NapA/BB690.

During the infectious cycle, $B$. burgdorferi survives in complex environments that require changes in gene expression to ensure survival and transmission. In response to the weak organic acid propionate, spirochetes initiate an acid stress response, promoting the expression of genes whose products can mitigate the detrimental effects of excess protons in the cytoplasm. Moreover, several monocarboxylic acids trigger the acid stress response that promotes $\mathrm{RpoN}$ - and RpoS-dependent virulence factor expression and a portion of the oxidative stress response regulon. It has long been supposed that $B$. burgdorferi does not have typical stress response mechanisms (Caimano et al., 2004); however, these data suggest that the acid stress response and virulence factor expression are likely linked, possibly to promote transmission or to facilitate long-term survival in the tick.

\section{Lactate and Acetate Are Present in the Tick Midgut Following Feeding}

Membrane-permeable acids can promote the expression of RpoSdependent virulence factors and trigger an acid stress response in B. burgdorferi. However, whether membrane-permeable acids are present throughout the infectious cycle is unknown. Although the biochemical composition of some relevant biological tissues where $B$. burgdorferi resides during the infectious cycle is known, the composition of the I. scapularis midgut is poorly defined. The membrane-permeable acids acetate, butyrate, and propionate have been identified in the midgut of spirochetecontaining arthropods (Odelson and Breznak, 1983; Gijzen and Barugahare, 1992) and bacteria identified in the tick midgut, including B. burgdorferi, produce membrane-permeable acids as metabolic end-products (Fraser et al., 1997; Narasimhan et al., 2014). Therefore, to assess the potential role for membranepermeable acids on signaling during the infectious cycle, we determined the concentration of membrane-permeable acids in the midgut of I. scapularis and in relevant biological fluids of host animals.

We measured the levels of monoprotic acids (lactate, acetate, propionate, butyrate, and benzoate), using reverse phase highpressure liquid chromatography (HPLC) and mass spectrometry (MS), in mouse sera, rabbit sera, and I. scapularis (B. burgdorferiinfected) midgut contents for the presence of these acids. Samples were isolated and processed as described previously and monocarboxylic acids were separated by HPLC and detected at $210 \mathrm{~nm}$ (Figures 5A-C). Peaks corresponding to acetate and lactate were identified based on their relative retention times compared to authentic standards (Figure 5D) and their identity was confirmed by MS. Mouse and rabbit sera had significant levels of lactate $(\sim 12.1 \pm 2.43$ and $\sim 6.7 \pm 0.17 \mathrm{mM}$, respectively) and acetate $(\sim 7.8 \pm 1.53$ and $\sim 10.6 \pm 0.3 \mathrm{mM}$, respectively). These two monoprotic acids were the predominant species detected in these sera samples. Midgut contents harvested from

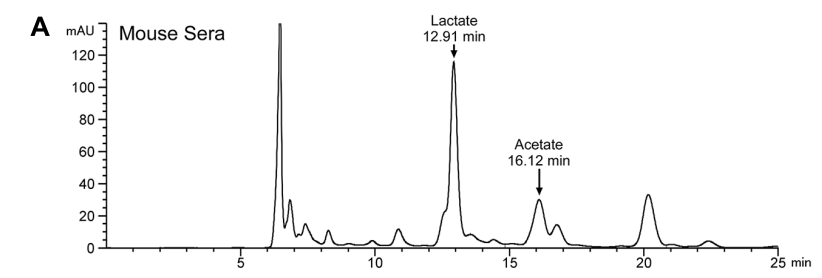

B
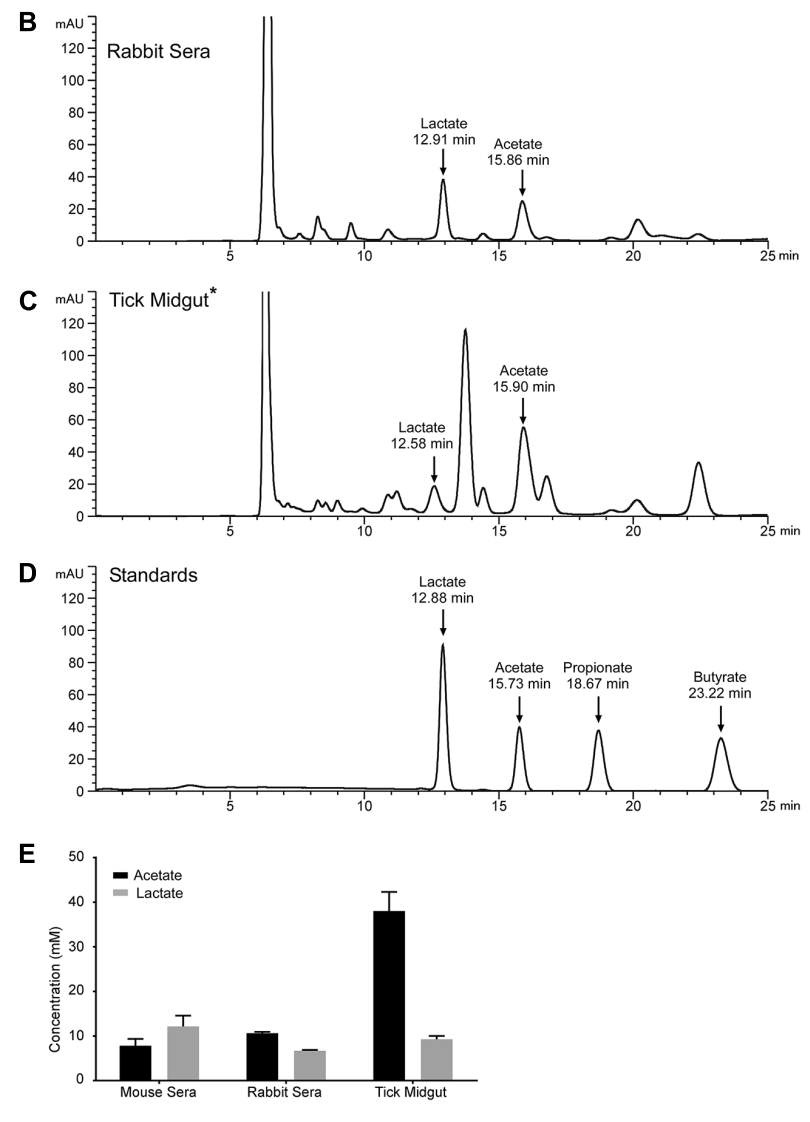

FIGURE 5 | Lactate and acetate are encountered by B. burgdorferi during the infectious cycle. Representative HPLC chromatograms monitoring absorbance at $210 \mathrm{~nm}$ of samples prepared from (A) mouse sera, (B) rabbit sera, (C) B. burgdorferi-infected tick midgut material, or (D) a standard containing lactate, acetate, propionate, and butyrate. Arrows indicate peaks confirmed by mass spectroscopy corresponding to lactate, acetate, propionate, and butyrate with their relative retention times listed in minutes. Asterisk indicates that the sample was diluted 1:1 with water prior to being run on the HPLC. Benzoate has a retention time of $30.1 \mathrm{~min}$ and is not displayed on this chromatogram. (E) Mean concentration of lactate or acetate found in mouse sera, rabbit sera, or the B. burgdorferi-infected tick midgut determined by HPLC analysis. Quantification of lactate and acetate was performed in biological triplicate for mouse sera and tick midgut contents. One rabbit blood sample was taken and sample preparation and HPLC/MS analysis was performed in technical triplicate.

replete, adult $I$. scapularis ticks that had fed upon the same rabbit whose sera we measured had a concentration of lactate $(\sim 9.3 \pm 0.8 \mathrm{mM})$ similar to levels measured in the rabbit sera (Figure 5E), while the acetate concentration was significantly higher $(38.0 \pm 4.3 \mathrm{mM})$. We were unable to detect butyrate, propionate, or benzoate in any of the samples analyzed. 
Initially, we had expected the levels of acetate and lactate to be increased in the tick midgut compared to levels initially detected in rabbit sera. That expectation was based upon the hypothesis that the removal of water from the blood meal, to facilitate saliva production in the feeding tick, would effectively increase the concentration of these acids in the midgut contents. While this might be the case for acetate, it appears that lactate, which is the end-product of $B$. burgdorferi metabolism is not increasing, suggesting that this acid might be consumed by the tick or by the resident midgut microbiota (Narasimhan et al., 2014).

Taken together, these data indicate that: (i) a weak organic acid could trigger a significant acid stress response in B. burgdorferi by affecting the intracellular $\mathrm{pH}$ of the cells, (ii) B. burgdorferi could mitigate these effects by generating or transporting neutralizing compounds (e.g., $\mathrm{NH}_{4}$, arginine) or "pumping" protons out of the cells $\left(\mathrm{V}_{\mathrm{o}} \mathrm{V}_{1}\right.$ ATPase), (iii) the acid stress response and virulence factor expression (e.g., OspC) was regulated by RpoN and RpoS, and (iv) acetate was present in the tick midgut at concentrations high enough to promote virulence factor expression via RpoSdependent gene regulation in vivo to possibly promote long-term survival in the tick midgut.

\section{DISCUSSION}

The effects of acid stress have been best characterized in enteric bacteria exposed to drastic changes in extracellular $\mathrm{pH}$ as they pass from the gastric stomach into the lower digestive tract. Despite the drastic change in $\mathrm{pH}$ due to concentrated $\mathrm{HCl}$, E. coli and Salmonella rebound very rapidly restoring $\mathrm{pH}$ homeostasis. To study the protective mechanisms that these bacteria use to defend against extreme $\mathrm{pH}$ changes, it is necessary to use monocarboxylic acids to dramatically extend the time it takes for the cytosol to rebound to $\mathrm{pH}$ homeostasis (Slonczewski et al., 2009). Protonated weak acids will diffuse through bacterial cell membranes, where the higher internal $\mathrm{pH}$ causes deprotonation that affects $\mathrm{pH}_{i}$ and the PMF. The concentration of the protonated acid is a function of the $\mathrm{p} K_{\mathrm{a}}$ of the acid and the $\mathrm{pH}$ of the solution. The closer the $\mathrm{pH}$ of the solution is to the $\mathrm{p} K_{\mathrm{a}}$ of the acid, the higher the proportion of protonated acid available. Additionally, in well-buffered solutions separated by a lipid bilayer, the rate of diffusion of weak acids across a membrane is increased, as the buffer increases the concentration of protonated acid species available (Gutknecht and Tosteson, 1973). The $\mathrm{p} K_{\mathrm{a}}$ of the acids used in our study suggest that there was a correlation between the proportion of protonated acids available and the level of expression of RpoS and RpoS-dependent factors. We found that the closer the $\mathrm{p} K_{\mathrm{a}}$ of the acid is to the $\mathrm{pH}$ of the media ( $\mathrm{pH} 7.0$ ), the greater the increase in RpoS expression (propionate $\mathrm{p} K_{\mathrm{a}}=4.88$, acetate $\mathrm{p} K_{\mathrm{a}}=4.76$, benzoate $\mathrm{p} K_{\mathrm{a}}=4.2$, lactate $\mathrm{p} K_{\mathrm{a}}=3.86$ ) (Figures 1C, 2B). However, benzoate did not follow this trend. Due to its non-polar ring structure, benzoate can also diffuse across a membrane in an unprotonated form and can act as an un-coupler of respiratory-chain-generated membrane potential, it is much more difficult for bacterial cells to reestablish $\mathrm{pH}$ homeostasis when exposed to this monocarboxylic acid. Moreover, propionate, unlike acetate (used in undecaprenyl-P synthesis) and lactate (used in anaerobic metabolism and secreted by BB0604), is not metabolized or actively transported/exported by $B$. burgdorferi. With no transporters or enzymes to eliminate benzoate or propionate, they likely accumulate in the cytoplasm, resulting in the greatest increase in expression of components of the Rrp2-RpoN-RpoS cascade. Additionally, induction of RpoS and OspC by weak organic acids seems limited to monocarboxylic acids as dicarboxylic acids, like succinate, failed to induce expression of OspC.

Neutralophiles, like B. burgdorferi, use a variety of mechanisms to deal with perturbations to intracellular $\mathrm{pH}$. Analysis of the B. burgdorferi genome identified genes encoding enzymes that have been previously shown in other bacteria to restore a favorable $\mathrm{pH}_{\mathrm{i}}$. These include: (i) putative coupled proton exchange with cations (BB0637-BB0638, BB0447, BB0724-BB0725, BB0380) and $\mathrm{Na}^{+} / \mathrm{H}^{+}$antiporters (BB0637BB0638); (ii) transporting neutralizing buffering molecules (polyamines: BB0639-BB0640); (iii) amino acid deaminases (BB0841-BB0843); (iv) and proton export via a $\mathrm{V}_{\mathrm{o}} \mathrm{V}_{1}$ vacuolartype ATPase (BB090-BB096). Cationic transport systems are highly conserved among bacteria and have been shown to act as primary proton pumps for restoring $\mathrm{pH}$ homeostasis in acidophiles, alkaliphiles as well as neutralophiles (Slonczewski et al., 2009). $\mathrm{Na}^{+} / \mathrm{H}^{+}$antiporters are also highly conserved among bacteria and are extremely important for restoring $\mathrm{pH}$ homeostasis in acidophiles and neutralophiles (Slonczewski et al., 2009). Indeed, these systems have been shown to be induced by a differential in $\mathrm{pH}_{\mathrm{i}}$ vs $\mathrm{pH}_{\mathrm{e}}$ and this seemed to be the case for these genes in B. burgdorferi (Foster, 2004; Slonczewski et al., 2009; Wilks et al., 2009). The role of the putative glutamate transporters (BB0401 and BB0729) in the acid stress response was difficult to understand since B. burgdorferi does not harbor the genes encoding any glutamate or lysine decarboxylases. These are the most common enzymes involved in restoring $\mathrm{pH}$ homeostasis in most neutralophiles. Additionally, BB0522 can potentially convert glutamate to glutamine in the presence of $\mathrm{NH}_{3}$ suggesting that glutamate may be used to generate glutamine which could act as an effective intracellular buffer. At this time, we do not have any experimental evidence that helps define the role of the glutamate transporters in the acid stress response. The arginine (ArcD/BB0843) transporter was also upregulated when $B$. burgdorferi cells are exposed to propionate and, more importantly, exogenous arginine could neutralize the effects of the strongest, non-metabolizable monocarboxylic acid tested (benzoate). ArcD putatively functions as an arginine/ornithine symport system. After transport, arginine can be hydrolyzed to citrulline $+\mathrm{NH}_{3}$ by ArcA and ultimately to ornithine + carbamoyl-P by ArcB/BB0842. Arginine and ornithine would be excellent cytosolic buffering molecules as would $\mathrm{NH}_{3}$. As important, $\mathrm{NH}_{3}$ which cannot be utilized in amino acid or nucleic acid biosynthetic pathways, could be conjugated to glutamate (forming glutamine) to be incorporated into newly synthesized proteins. The induction of the genes encoding the $\mathrm{V}_{\mathrm{o}} \mathrm{V}_{1}$ vacuolar ATPase during acid stress was fascinating and, in retrospect, predictable. Without respiratory enzyme complexes, $B$. burgdorferi utilizes this ATPase to hydrolyze ATP and pump protons across the cytoplasmic 
membrane to maintain the PMF which is required for motility and energizes critical transport systems. It seemed very likely that the vacuolar ATPase was a major defense against acid/proton stress. It should be noted that the lack of induction of some of the cation transport systems does not mean that they do not play a role in restoring $\mathrm{pH}$ homeostasis. It has been shown that constitutive expression of these systems is adequate to protect bacteria from acid stress (Foster, 1999; Slonczewski et al., 2009). What seems clear is that $B$. burgdorferi makes use of a unique combination of enzymes to maintain $\mathrm{pH}$ homeostasis that are specifically tailored to the environments in which it survives.

To sense its environment, B. burgdorferi harbors the genes encoding only two two-component regulatory systems: Hk1Rrp1 which control the secondary messenger, cyclic-di-GMP; and Hk2-Rrp2 which, through this RpoN-specific response regulator, controls the transcription of RpoS (Samuels, 2011). Unlike more canonical response regulators, Rrp2 lacks a transmembrane domain and cannot interact directly with extracellular ligands (Yang et al., 2003). How Rrp2 senses environmental signals and translates those into appropriate changes in gene expression remains to be elucidated. Our data demonstrated that a decrease in $\mathrm{pH}_{\mathrm{i}}$ promoted the transcription of RpoNand RpoS-dependent genes, indicating that the Rrp2-RpoNRpoS signaling cascade was activated under these conditions (Xu et al., 2010; Richards et al., 2015). Additionally, when we neutralized the acidification of the cytoplasm, we prevented the activation of the Rrp2-RpoN-RpoS cascade (Figures 4A-C). Changes in intracellular $\mathrm{pH}$ can affect multiple cellular functions simultaneously, including enzyme function and disruption of the PMF. In B. burgdorferi, with few proton-pumping transporters and lacking respiratory enzymes, the $\mathrm{pH}_{\mathrm{i}}$ and the PMF are inextricably linked. Therefore, a weak acid mediated decrease in $\mathrm{pH}_{\mathrm{i}}$ or disruption of the PMF, could be an intracellular signal that reflects changes in the extracellular environment. Moreover, a decrease in $\mathrm{pH}_{\mathrm{i}}$ or disruption of the PMF likely results in a decrease in the intracellular concentration of ATP as the vacuolar ATPase hydrolyzes ATP to export protons to maintain the PMF and restore $\mathrm{pH}$ homeostasis. This decrease in the intracellular energy pool could also be an activation signal for the Rrp2RpoN-RpoS pathway. Currently, the vacuolar ATPase is thought to be the major contributor to generating the PMF (Radolf and Samuels, 2010), making it difficult to separate the PMF from the vacuolar ATPase activity in B. burgdorferi. Further mechanistic studies to determine the function of Rrp2 should help elucidate how perturbations to $\mathrm{pH}$ homeostasis activate this pathway.

Whether membrane-permeable acids found in the I. scapularis midgut help promote the transmission of $B$. burgdorferi by triggering the expression of virulence factors is unresolved, however, seems unlikely. The monocarboxylic acids acetate and lactate were detected at significant levels late in the feeding cycle, well after the expression of key virulence factors and the initiation of transmission have begun (Schwan and Piesman, 2000). It is more likely that membrane-permeable acids elicit an acid stress response in B. burgdorferi to protect spirochetes from multiple stressors found in the tick midgut (e.g., RNS and ROS) and promote long-term survival. The conditions found in the I. scapularis midgut, both physiological and biochemical, during and following blood feeding, are likely critical to pathogen transmission and long-term survival.

The tick midgut and its contents are a complex, constantly changing biome in which many conditions, macromolecules and bioactive compounds remain undefined (Yang et al., 2000; Bontemps-Gallo et al., 2016). We now report millimolar concentrations of both lactate and acetate in the midgut of replete ticks (Figure 5E). Lactate levels were lower than concentrations measured in rabbit or mouse sera while the concentration of acetate was significantly higher than those measured in animal sera (Figure 5E). This result was intriguing since the acetate concentration increased sharply between rabbit sera and the tick midgut contents while lactate levels stayed relatively constant (Figure 5E). If the levels of the organic acids detected in the midgut were the result of only the concentration of the blood meal, we would expect lactate and acetate to increase proportionally. Possibly, the observed lactate levels reflect the utilization of this acid by the tick and/or the midgut microbiome at rates exceeding the contribution of the incoming blood or the metabolism of lactate producers like B. burgdorferi. In the tick midgut, the concentration of lactate was lower than what we used in our experiments ( $20 \mathrm{mM}$ lactate vs $9 \mathrm{mM}$ in the midgut) while the acetate concentration was detected in the midgut at a higher concentration than what we used in our experiments (20 $\mathrm{mM}$ acetate vs $38 \mathrm{mM}$ in the midgut). Interestingly, even when supplementing $20 \mathrm{mM}$ acetate in the media, RpoS and OspC expression are markedly increased. Furthermore, RpoS and $\mathrm{OspC}$ expression increase in a concentration dependent manner when exposed to acetate, with robust expression at acetate concentrations close to those we report in the tick midgut (30 $\mathrm{mM}$ acetate vs $35 \mathrm{mM}$ in the midgut) (Xu et al., 2010; Richards et al., 2015).

Currently, we do not know the source of the acetate in the tick midgut and several possible factors may be affecting the acetate concentration we observed. First, these levels may reflect the concentration of a non-utilizable blood component. It seems unlikely that this is the sole explanation considering the dynamic nature of the environment in a feeding tick midgut. Secondly, the midgut microbiota may be contributing acetate generated as a product of microbial metabolism. Microbes present in some arthropods can carry out acetogenesis $\left(\mathrm{H}_{2}+\mathrm{CO}_{2} \rightarrow\right.$ acetate), generating significant amounts of acetate in the gut, which is absorbed by the gut cells and utilized as an energy source (Breznak and Switzer, 1986; Leadbetter et al., 1999). There is no indication that microbes in the tick midgut can perform acetogenesis or that ticks absorb acetate from the midgut. However, the conversion of lactate to acetate seems likely considering the composition of the midgut microflora and that a decrease in the complexity of the tick midgut microbiome has adverse effects on tick physiology (Narasimhan et al., 2014). While these factors may influence the overall observed acetate concentration in the tick midgut, what is clear is that the midgut biochemistry and physiology are complex and we are just beginning to dissect this extremely dynamic environment.

Our study identified an acid stress response in B. burgdorferi that activated the Rrp2-RpoN-RpoS signal transduction cascade, some BosR-directed oxidative stress response genes, and 
increased the expression of genes encoding proteins involved in restoring $\mathrm{pH}$ homeostasis. An increase in the transcription of these genes was stimulated by membrane-permeable monocarboxylic acids, some of which (acetate and lactate) were identified in the tick midgut following feeding. Moreover, we have now demonstrated that perturbations in $\mathrm{pH}$ homeostasis activated the Rrp2-RpoN-RpoS/BosR regulatory cascade(s) that was previously attributed to Acetyl-P (Xu et al., 2010). We did not identify the intracellular signal that ultimately up regulates these pathways, however, the effects of the decrease in $\mathrm{pH}_{\mathrm{i}}$ on the vacuolar ATPase might suggest that ATP or GTP may serve as effective intracellular energy "sensors." While it seemed clear that the acid stress response and $\mathrm{pH}_{\mathrm{i}}$ effected virulence factor expression in B. burgdorferi in vitro, it seemed unlikely that these parameters affect transmission in vivo. Since the expression of virulence factors, like OspC, are so tightly linked to RpoS, in vitro conditions that trigger Rrp2-RpoN-RpoS and BosR-dependent gene regulation do not necessarily indicate that these conditions are required for, or play a role in, successful transmission. Considering the complexity of the tick midgut, it seems likely that multiple factors are required to maximize and synchronize the expression of virulence factors to the tick feeding cycle to promote successful transmission.

\section{MATERIALS AND METHODS}

\section{Borrelia burgdorferi Strains and Growth Conditions}

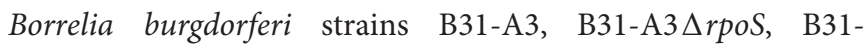
$\mathrm{A} 3 \Delta r p o N, \mathrm{~B} 31-\mathrm{A} 3 \Delta b o s R-\mathrm{L} 9$, and $\mathrm{B} 31-\mathrm{A} 3 \Delta h k 2$ have been described previously (Elias et al., 2000; Fisher et al., 2005; $\mathrm{Xu}$ et al., 2010; Katona, 2015). Membrane-permeable acids were tested as previously described (Wilks and Slonczewski, 2007) with minor modifications. B. burgdorferi strains were grown at $34^{\circ} \mathrm{C}$ in BSK-II medium $\mathrm{pH} 7.0$ under microaerobic conditions $\left(3-5 \% \mathrm{O}_{2}\right)$ to mid-logarithmic phase $\left(5-7 \times 10^{7} / \mathrm{mL}\right)$. Spirochetes were subsequently diluted to $1 \times 10^{6} / \mathrm{mL}$ in BSK-II medium pH 7.0 or BSK-II medium pH 7.0 supplemented with $20 \mathrm{mM}$ sodium acetate, sodium benzoate, sodium lactate, sodium propionate or in combination with $20 \mathrm{mM}$ L-arginine or L-alanine (Sigma-Aldrich, St. Louis, MO, United States).

\section{SDS-PAGE and Immunoblotting}

Spirochetes were grown to $1-5 \times 10^{7} / \mathrm{mL}$, harvested by centrifugation, washed twice with HEPES-NaCl buffer $(20 \mathrm{mM}$ HEPES, pH 8.0; $50 \mathrm{mM} \mathrm{NaCl})(\mathrm{HN})$ and whole cell lysates were analyzed by SDS-PAGE and immunoblots as previously described (Dulebohn et al., 2014). Antibodies were used at the following concentrations: $\alpha$ OspC 1:1000 (Tilly et al., 2007), $\alpha$ BBA66 1:4000 (Clifton et al., 2006), $\alpha$ NapA/BB690 1:5000 (Boylan et al., 2003), $\alpha$ RpoS 1:500 (Dulebohn et al., 2014), $\alpha$ Flagellin 1:100 (Barbour et al., 1986), and $\alpha$ Fur/BosR 1:1000 (Boylan et al., 2003). Immunoblots were incubated in $1 \times$ TBS-T with an HRP (horseradish peroxidase) conjugated recombinant protein A (Thermo Fisher Scientific, Grand Island, NY, United States) at 1:4000 for $1 \mathrm{~h}$ at RT with shaking for the detection of OspC,
NapA/BB690, RpoS, and BosR. For detection of flagellin, an antimouse secondary antibody-HRP conjugated antibody was used at 1:10,000 (Thermo Fisher Scientific) and detection of BBA66 was performed with a secondary HRP conjugated $\alpha$ Chicken antibody at 1:50,000 in $1 \times$ TBS-T as previously described (Clifton et al., 2006). Immunoblots were developed using SuperSignal West Pico chemiluminescent substrate kit (Thermo Fisher Scientific) and X-ray film (Lab Scientific Inc., Livingston, NJ, United States). Quantification of OspC and BBA66 (Supplementary Figure S1) was performed using Image J software (Schneider et al., 2012).

\section{Quantitative Reverse Transcriptase PCR}

qRT-PCR was performed as previously described (Dulebohn et al., 2014) with minor modifications. Briefly, spirochetes were grown in BSK-II medium with or without benzoate or propionate, harvested at $1-5 \times 10^{7} / \mathrm{mL}$, and washed with $\mathrm{HN}$ buffer. Spirochetes were resuspended in $1 \mathrm{~mL}$ of TRI reagent (Sigma-Aldrich) and RNA harvested using a Direct-zol RNA mini prep kit (ZYMO Research, Irvine, CA, United States). RNA ( $5 \mu \mathrm{g}$ ) was treated for $1 \mathrm{~h}$ with Turbo DNase (Thermo Fisher Scientific) and $1 \mathrm{ng}$ subsequently used for cDNA synthesis using High-Capacity cDNA Reverse Transcription Kit (Thermo Fisher Scientific). cDNA was then used in qPCR reactions using the $5^{\prime}$ nuclease Prime Time Assay from IDT on a Viia 7 instrument (Thermo Fisher Scientific). Primers and probes are listed in Supplementary Table S1 and statistical analysis done with GraphPad PRISM software (GraphPad Software Inc., La Jolla, CA, United States).

\section{$\beta$-Galactosidase Activity Assays}

Analysis of $\beta$-galactosidase activity was performed as previously described (Dulebohn et al., 2014). Briefly, B. burgdorferi strain B31-A3 containing the pBHospCp-lac $Z_{\mathrm{Bb}}$ (Hayes et al., 2010) or pBSV2G-rpoS $\mathrm{p}_{92}-$ lac $_{B b}$ shuttle vector, harboring the ospC or $r p o S$ promoter, respectively, were grown to $1-5 \times 10^{7}$ spirochetes $/ \mathrm{mL}$ as stated above and $\beta$-galactosidase activity was assayed in three biological replicates as previously described (Dulebohn et al., 2014). The pBSV2G-rpoSp $92-$ lac $_{\mathrm{Bb}}$ shuttle vector was constructed by amplifying the $r p o S$ promoter from B. burgdorferi B31-A3 gDNA using primers rpoSP92-F and rpoSP-R (Supplementary Table S1) and the 92 bp fragment ( $r p o S p_{92}$ ) was ligated immediately upstream of the $l a c Z_{\mathrm{Bb}}$ gene in $\mathrm{pBH}_{\text {Bac }} Z_{\mathrm{Bb}}$ (Hayes et al., 2010). Relevant sequences were confirmed by sequencing.

\section{Cytoplasmic pH Analysis with Membrane-Permeable pH Sensitive Dye}

Borrelia burgdorferi strain B31-A3 was grown to $1-2 \times 10^{7}$ spirochetes per $\mathrm{mL}$ in BSK-II ( $\mathrm{pH}$ 7.0) and cultures were split and treated with either sodium acetate, sodium lactate, sodium propionate, sodium benzoate or a combination of sodium benzoate and arginine, or sodium benzoate and alanine $(20 \mathrm{mM}$ final concentration of each). Cultures were then incubated at $35^{\circ} \mathrm{C}$ for $48 \mathrm{~h}$ under microaerobic conditions $\left(3-5 \% \quad \mathrm{O}_{2}\right)$ and equivalent numbers of spirochetes $\left(3 \times 10^{9}\right.$ spirochetes $)$ were harvested 
by centrifugation at $3,000 \mathrm{rpm}$ for $10 \mathrm{~min}$ and washed twice with Live Cell Imaging Solution (LCIS) (Thermo Fisher Scientific). pHrodo Green AM (Thermo Fisher Scientific) was then used according to the manufacturer's instructions, samples incubated at $37^{\circ} \mathrm{C}$ for $30 \mathrm{~min}$ and then washed twice with LCIS. Fluorescence was quantified using a BioTek synergy spectrophotometer (BioTek, Winooski, VT, United States) using Excitation/Emission filters of $485 \mathrm{~nm} / 560 \mathrm{~nm}$, respectively. At least two independent biological replicates were performed for each condition tested and each biological replicate was analyzed in duplicate. Extended imaging led to increased background fluorescence so ReadyProbes ${ }^{\text {TM }}$ Backdrop ${ }^{\circledR}$ Green Background Suppressor reagent (Thermo Fisher Scientific) was added to each sample and then imaged on a Nikon Eclipse E80 epifluorescence microscope $(100 \times$ magnification, CY3 filter). All images were captured with identical exposure times (1 s).

\section{Analysis of Biological Samples by High-Pressure Liquid Chromatography and Mass Spectrometry}

Mouse infection and tick feeding were performed as previously described (Bontemps-Gallo et al., 2016). Briefly, laboratoryraised I. scapularis larvae (Oklahoma State University) were fed to repletion on B. burgdorferi B31-A3 infected mice, collected and allowed to molt to nymphs. Nymphs were then fed to repletion on naïve RML mice, collected and allowed to molt into adults. Adult ticks were then fed to repletion on New Zealand White rabbits. Midgut material was collected as previously described (Bontemps-Gallo et al., 2016) and diluted 1:2 in sterile water. Blood samples from New Zealand White rabbits or RML mice were clarified by centrifugation at $2000 \times g$ for $10 \mathrm{~min}$ and the serum fraction recovered. All samples were cleared by centrifugation using a Microcon-10 (EMD Millipore, Billerica, MA, United States) at $10,000 \times g$ until completion. The filtrate was then analyzed using an Agilent 1200 Series liquid chromatography system coupled to a 6120 Series Single Quad mass spectrometer equipped with an ESI source (Agilent Technologies, Santa Clara, CA, United States). Twenty microliters of sample or standard was injected and separated on an Aminex HPX-87 $300 \mathrm{~mm} \times 7.8 \mathrm{~mm}$ column (BioRad, Hercules, CA, United States) following the manufacturers protocol. Absorbance data was collected from 200 to $750 \mathrm{~nm}$ to monitor $\mathrm{COOH}$ groups at $210 \mathrm{~nm}$ and aid in compound identification and peak purity analysis. Negative ion mass data was acquired as previously described (Ibanez and Bauer, 2014), with a $350^{\circ} \mathrm{C}$ nitrogen gas temperature and select ion monitoring for the $[\mathrm{M}-\mathrm{H}]^{-}$of acetic acid (59.00), lactic acid (89.1), propionic acid (73.1), and butyric acid (88.1). The mass spectrometer was tuned to the following internal mass reference ions $(\mathrm{m} / \mathrm{z})$ : $112.99,601.98,1033.99$, and 1633.95. Sample identifications were made by retention time relative to a standard and confirmed by MS. Quantification of lactate and acetate was performed with biological triplicates for mouse sera and tick midgut contents. One rabbit blood sample was taken and sample preparation and HPLC/MS analysis was performed in triplicate. Quantification of lactate and acetate was performed by monitoring UV absorbance at $210 \mathrm{~nm}$ and calculating the area under the peak relative to a standard curve for each compound.

\section{ETHICS STATEMENT}

Mouse infection studies were carried out in accordance with the Animal Welfare Act (AWA 1990), the guidelines of the National Institutes of Health, Public Health Service Policy on Humane Care (PHS 2002) and Use of Laboratory Animals and the United States Institute of Laboratory Animal Resources, National Research Council, Guide for the Care and Use of Laboratory Animals. All animal work was done according to protocols approved by the Rocky Mountain Laboratories, NIAID, NIH Animal Care and Use Committee (Protocol Number 2014021). The Rocky Mountain Laboratories are accredited by the International Association for Assessment and Accreditation of Laboratory Animal Care (AAALAC).

\section{AUTHOR CONTRIBUTIONS}

DD designed and performed the experiments, analyzed the data, and wrote the manuscript; CR, HS, and KL designed and performed the experiments; FG conceived the project, provided reagents and conceptual design, and wrote the manuscript.

\section{FUNDING}

This study was supported by the Intramural Research Program of the National Institute of Allergy and Infectious Diseases, National Institutes of Health. The funders were not involved in the study design, data collection and analysis, decision to publish, or preparation of the manuscript.

\section{ACKNOWLEDGMENTS}

We would like to thank Dr. Patricia Rosa for the use of her fluorescence microscope and $\alpha$-OspC antisera and Dr. James Carroll for $\alpha$-BBA66 antisera. We would also like to thank Drs. Jorge Benach and Frank Yang for B31-A3 $\Delta$ bosR and B31A $3 \Delta h k 2$ strains, respectively. We thank Dr. Tom Schwan for the use of his fluorescence microscope. We are also grateful to Anita Mora for help with figure preparation. We thank Dr. Philip Stewart, Dr. Joe Hinnebusch, and all the members of the Gherardini lab for their critical review of the manuscript.

\section{SUPPLEMENTARY MATERIAL}

The Supplementary Material for this article can be found online at: http://journal.frontiersin.org/article/10.3389/fmicb. 2017.01734/full\#supplementary-material 


\section{REFERENCES}

Barbour, A. G., Hayes, S. F., Heiland, R. A., Schrumpf, M. E., and Tessier, S. L. (1986). A Borrelia-specific monoclonal antibody binds to a flagellar epitope. Infect. Immun. 52, 549-554.

Bearson, S., Bearson, B., and Foster, J. W. (1997). Acid stress responses in enterobacteria. FEMS Microbiol. Lett. 147, 173-180. doi: 10.1111/j.1574-6968. 1997.tb10238.x

Bontemps-Gallo, S., Lawrence, K., and Gherardini, F. C. (2016). Two different virulence-related regulatory pathways in Borrelia burgdorferi are directly affected by osmotic fluxes in the blood meal of feeding Ixodes ticks. PLOS Pathog. 12:e1005791. doi: 10.1371/journal.ppat.1005791

Bourret, T. J., Lawrence, K. A., Shaw, J. A., Lin, T., Norris, S. J., and Gherardini, F. C. (2016). The nucleotide excision repair pathway protects Borrelia burgdorferi from nitrosative stress in Ixodes scapularis ticks. Front. Microbiol. 7:1397. doi: 10.3389/fmicb.2016.01397

Boylan, J. A., Hummel, C. S., Benoit, S., Garcia-Lara, J., Treglown-Downey, J., Crane, E. J. 3rd., et al. (2006). Borrelia burgdorferi bb0728 encodes a coenzyme A disulphide reductase whose function suggests a role in intracellular redox and the oxidative stress response. Mol. Microbiol. 59, 475-486. doi: 10.1111/j.13652958.2005.04963.x

Boylan, J. A., Posey, J. E., and Gherardini, F. C. (2003). Borrelia oxidative stress response regulator, BosR: a distinctive $\mathrm{Zn}$-dependent transcriptional activator. Proc. Natl. Acad. Sci. U.S.A. 100, 11684-11689. doi: 10.1073/pnas.2032956100

Breznak, J. A., and Switzer, J. M. (1986). Acetate synthesis from $\mathrm{H}_{2}$ plus $\mathrm{CO}_{2}$ by termite gut microbes. Appl. Environ. Microbiol. 52, 623-630.

Burgdorfer, W., Barbour, A. G., Hayes, S. F., Benach, J. L., Grunwaldt, E., and Davis, J. P. (1982). Lyme disease - a tick-borne spirochetosis? Science 216, 1317-1319.

Burtnick, M. N., Downey, J. S., Brett, P. J., Boylan, J. A., Frye, J. G., Hoover, T. R., et al. (2007). Insights into the complex regulation of rpoS in Borrelia burgdorferi. Mol. Microbiol. 65, 277-293. doi: 10.1111/j.1365-2958.2007.05813.x

Caimano, M. J., Eggers, C. H., Hazlett, K. R., and Radolf, J. D. (2004). RpoS is not central to the general stress response in Borrelia burgdorferi but does control expression of one or more essential virulence determinants. Infect. Immun. 72, 6433-6445. doi: 10.1128/IAI.72.11.6433-6445.2004

Caimano, M. J., Iyer, R., Eggers, C. H., Gonzalez, C., Morton, E. A., Gilbert, M. A., et al. (2007). Analysis of the RpoS regulon in Borrelia burgdorferi in response to mammalian host signals provides insight into RpoS function during the enzootic cycle. Mol. Microbiol. 65, 1193-1217. doi: 10.1111/j.1365-2958.2007. 05860.x

Carroll, J. A., Garon, C. F., and Schwan, T. G. (1999). Effects of environmental pH on membrane proteins in Borrelia burgdorferi. Infect. Immun. 67, 3181-3187.

Clifton, D. R., Nolder, C. L., Hughes, J. L., Nowalk, A. J., and Carroll, J. A. (2006) Regulation and expression of bba66 encoding an immunogenic infectionassociated lipoprotein in Borrelia burgdorferi. Mol. Microbiol. 61, 243-258. doi: 10.1111/j.1365-2958.2006.05224.x

Cusumano, Z. T., and Caparon, M. G. (2015). Citrulline protects Streptococcus pyogenes from acid stress using the arginine deiminase pathway and the $\mathrm{F}_{1} \mathrm{~F}_{\mathrm{o}}$ ATPase. J. Bacteriol. 197, 1288-1296. doi: 10.1128/JB.02517-14

Dulebohn, D. P., Hayes, B. M., and Rosa, P. A. (2014). Global repression of hostassociated genes of the Lyme disease spirochete through post-transcriptional modulation of the alternative sigma factor RpoS. PLOS ONE 9:e93141. doi: 10.1371/journal.pone.0093141

Dunham-Ems, S. M., Caimano, M. J., Eggers, C. H., and Radolf, J. D. (2012). Borrelia burgdorferi requires the alternative sigma factor RpoS for dissemination within the vector during tick-to-mammal transmission. PLOS Pathog. 8:e1002532. doi: 10.1371/journal.ppat.1002532

Eggers, C. H., Caimano, M. J., Malizia, R. A., Kariu, T., Cusack, B., Desrosiers, D. C., et al. (2011). The coenzyme A disulphide reductase of Borrelia burgdorferi is important for rapid growth throughout the enzootic cycle and essential for infection of the mammalian host. Mol. Microbiol. 82, 679-697. doi: 10.1111/j. 1365-2958.2011.07845.x

Elias, A. F., Bono, J. L., Carroll, J. A., Stewart, P., Tilly, K., and Rosa, P. (2000). Altered stationary phase response in a Borrelia burgdorferi rpoS mutant. J. Bacteriol. 182, 2909-2918. doi: 10.1128/JB.182.10.2909-2918.2000

Esteve-Gassent, M. D., Elliott, N. L., and Seshu, J. (2009). sodA is essential for virulence of Borrelia burgdorferi in the murine model of Lyme disease. Mol. Microbiol. 71, 594-612. doi: 10.1111/j.1365-2958.2008.06549.x
Fisher, M. A., Grimm, D., Henion, A. K., Elias, A. F., Stewart, P. E., Rosa, P. A., et al. (2005). Borrelia burgdorferi sigma54 is required for mammalian infection and vector transmission but not for tick colonization. Proc. Natl. Acad. Sci. U.S.A. 102, 5162-5167. doi: 10.1073/pnas.0408536102

Foster, J. W. (1999). When protons attack: microbial strategies of acid adaptation. Curr. Opin. Microbiol. 2, 170-174. doi: 10.1016/S1369-5274(99) 80030-7

Foster, J. W. (2004). Escherichia coli acid resistance: tales of an amateur acidophile. Nat. Rev. Microbiol. 2, 898-907. doi: 10.1038/nrmicro1021

Fraser, C. M., Casjens, S., Huang, W. M., Sutton, G. G., Clayton, R., Lathigra, R., et al. (1997). Genomic sequence of a Lyme disease spirochaete, Borrelia burgdorferi. Nature 390, 580-586. doi: 10.1038/37551

Gijzen, H. J., and Barugahare, M. (1992). Contribution of anaerobic protozoa and methanogens to hindgut metabolic activities of the American cockroach, Periplaneta americana. Appl. Environ. Microbiol. 58, 2565-2570.

Groshong, A. M., Gibbons, N. E., Yang, X. F., and Blevins, J. S. (2012). Rrp2, a prokaryotic enhancer-like binding protein, is essential for viability of Borrelia burgdorferi. J. Bacteriol. 194, 3336-3342. doi: 10.1128/JB.00253-12

Gutknecht, J., and Tosteson, D. C. (1973). Diffusion of weak acids across lipid bilayer membranes: effects of chemical reactions in the unstirred layers. Science 182, 1258-1261. doi: 10.1126/science.182.4118.1258

Hayes, B., Jewett, M., and Rosa, P. (2010). A lacZ reporter system for use in Borrelia burgdorferi. Appl. Environ. Microbiol. 76, 7407-7412. doi: 10.1128/AEM. 01389-10

Hübner, A., Wang, X., Nolen, D. M., Popova, T. G., Cabello, F. C., and Norgard, M. (2001). Expression of Borrelia burgdorferi OspC and DbpA is controlled by a RpoN-RpoS regulatory pathway. Proc. Natl. Acad. Sci. U.S.A. 98, 12724-12729. doi: $10.1073 /$ pnas. 231442498

Hyde, J. A., Seshu, J., and Skare, J. T. (2006). Transcriptional profiling of Borrelia burgdorferi containing a unique bos $R$ allele identifies a putative oxidative stress regulon. Microbiology 152, 2599-2609. doi: 10.1099/mic.0.28996-0

Hyde, J. A., Shaw, D. K., Smith Iii, R., Trzeciakowski, J. P., and Skare, J. T. (2009). The BosR regulatory protein of Borrelia burgdorferi interfaces with the RpoS regulatory pathway and modulates both the oxidative stress response and pathogenic properties of the Lyme disease spirochete. Mol. Microbiol. 74, 1344-1355. doi: 10.1111/j.1365-2958.2009.06951.x

Ibanez, A. B., and Bauer, S. (2014). Analytical method for the determination of organic acids in dilute acid pretreated biomass hydrolysate by liquid chromatography-time-of-flight mass spectrometry. Biotechnol. Biofuels 7:145. doi: 10.1186/s13068-014-0145-3

Kanjee, U., and Houry, W. A. (2013). Mechanisms of acid resistance in Escherichia coli. Annu. Rev. Microbiol. 67, 65-81. doi: 10.1146/annurev-micro-092412155708

Katona, L. I. (2015). The Fur homologue BosR requires Arg39 to activate rpoS transcription in Borrelia burgdorferi and thereby direct spirochaete infection in mice. Microbiology 161, 2243-2255. doi: 10.1099/mic.0.000166

Kirkpatrick, C., Maurer, L. M., Oyelakin, N. E., Yoncheva, Y. N., Maurer, R., and Slonczewski, J. L. (2001). Acetate and formate stress: opposite responses in the proteome of Escherichia coli. J. Bacteriol. 183, 6466-6477. doi: 10.1128/JB.183. 21.6466-6477.2001

Lane, R. S., Piesman, J., and Burgdorfer, W. (1991). Lyme borreliosis: relation of its causative agent to its vectors and hosts in North America and Europe. Annu. Rev. Entomol. 36, 587-609. doi: 10.1146/annurev.en.36.010191.003103

Leadbetter, J. R., Schmidt, T. M., Graber, J. R., and Breznak, J. A. (1999). Acetogenesis from $\mathrm{H} 2$ plus $\mathrm{CO} 2$ by spirochetes from termite guts. Science 283, 686-689. doi: 10.1126/science.283.5402.686

Lybecker, M. C., and Samuels, D. S. (2007). Temperature-induced regulation of RpoS by a small Rna in Borrelia burgdorferi. Mol. Microbiol. 64, 1075-1089. doi: 10.1111/j.1365-2958.2007.05716.x

Marquis, R. E., Bender, G. R., Murray, D. R., and Wong, A. (1987). Arginine deiminase system and bacterial adaptation to acid environments. Appl. Environ. Microbiol. 53, 198-200.

Miksa, M., Komura, H., Wu, R., Shah, K. G., and Wang, P. (2009). A novel method to determine the engulfment of apoptotic cells by macrophages using pHrodo succinimidyl ester. J. Immunol. Methods 342, 71-77. doi: 10.1016/j.jim.2008. 11.019

Mindell, J. A. (2012). Lysosomal acidification mechanisms. Annu. Rev. Physiol. 74, 69-86. doi: 10.1146/annurev-physiol-012110-142317 
Narasimhan, S., Rajeevan, N., Liu, L., Zhao, Y. O., Heisig, J., Pan, J., et al. (2014). Gut microbiota of the tick vector Ixodes scapularis modulate colonization of the Lyme disease spirochete. Cell Host Microbe 15, 58-71. doi: 10.1016/j.chom. 2013.12.001

Nelder, M. P., Russell, C. B., Sheehan, N. J., Sander, B., Moore, S., Li, Y., et al. (2016). Human pathogens associated with the blacklegged tick Ixodes scapularis: a systematic review. Parasit. Vectors 9:265. doi: 10.1186/s13071-016-1529-y

Nishi, T., and Forgac, M. (2002). The vacuolar (H+)-ATPases-nature's most versatile proton pumps. Nat. Rev. Mol. Cell Biol. 3, 94-103. doi: 10.1038/nrm729

Odelson, D. A., and Breznak, J. A. (1983). Volatile Fatty Acid production by the hindgut microbiota of xylophagous termites. Appl. Environ. Microbiol. 45, 1602-1613.

Ouyang, Z., Kumar, M., Kariu, T., Haq, S., Goldberg, M., Pal, U., et al. (2009). BosR (BB0647) governs virulence expression in Borrelia burgdorferi. Mol. Microbiol. 74, 1331-1343. doi: 10.1111/j.1365-2958.2009.06945.x

Radolf, J. D., and Samuels, D. S. (2010). Borrelia: Molecular Biology, Host Interaction and Pathogenesis. Norfolk: Caister Academic Press.

Ramsey, M. E., Hyde, J. A., Medina-Perez, D. N., Lin, T., Gao, L., Lundt, M. E., et al. (2017). A high-throughput genetic screen identifies previously uncharacterized Borrelia burgdorferi genes important for resistance against reactive oxygen and nitrogen species. PLOS Pathog. 13:e1006225. doi: 10.1371/journal.ppat. 1006225

Richards, C. L., Lawrence, K. A., Su, H., Yang, Y., Yang, X. F., Dulebohn, D. P., et al. (2015). Acetyl-phosphate is not a global regulatory bridge between virulence and central metabolism in Borrelia burgdorferi. PLOS ONE 10:e0144472. doi: 10.1371/journal.pone.0144472

Ryan, S., Begley, M., Gahan, C. G., and Hill, C. (2009). Molecular characterization of the arginine deiminase system in Listeria monocytogenes: regulation and role in acid tolerance. Environ. Microbiol. 11, 432-445. doi: 10.1111/j.1462-2920. 2008.01782.x

Samuels, D. S. (2011). Gene regulation in Borrelia burgdorferi. Annu. Rev. Microbiol. 65, 479-499. doi: 10.1146/annurev.micro.112408.134040

Schneider, C. A., Rasband, W. S., and Eliceiri, K. W. (2012). NIH Image to ImageJ: 25 years of image analysis. Nat. Methods 9, 671-675. doi: 10.1038/nmeth.2089

Schwan, T. G., and Piesman, J. (2000). Temporal changes in outer surface proteins $\mathrm{A}$ and $\mathrm{C}$ of the Lyme disease-associated spirochete, Borrelia burgdorferi, during the chain of infection in ticks and mice. J. Clin. Microbiol. 39, 382-388.

Schwan, T. G., Piesman, J., Golde, W. T., Dolan, M. C., and Rosa, P. A. (1995). Induction of an outer surface protein on Borrelia burgdorferi during tick feeding. Proc. Natl. Acad. Sci. U.S.A. 92, 2909-2913. doi: 10.1073/pnas.92. 7.2909
Slonczewski, J. L., Fujisawa, M., Dopson, M., and Krulwich, T. A. (2009). Cytoplasmic pH measurement and homeostasis in bacteria and archaea. $A d v$. Microb. Physiol. 55, 1-79, 317. doi: 10.1016/S0065-2911(09)05501-5

Steere, A. C., Grodzicki, R. L., Kornblatt, A. N., Craft, J. E., Barbour, A. G., Burgdorfer, W., et al. (1983). The spirochetal etiology of Lyme disease. N. Engl. J. Med. 308, 733-740. doi: 10.1056/NEJM198303313081301

Tilly, K., Bestor, A., Jewett, M. W., and Rosa, P. (2007). Rapid clearance of Lyme disease spirochetes lacking OspC from skin. Infect. Immun. 75, 1517-1519. doi: 10.1128/IAI.01725-06

Van Laar, T. A., Lin, Y. H., Miller, C. L., Karna, S. L., Chambers, J. P., and Seshu, J. (2012). Effect of levels of acetate on the mevalonate pathway of Borrelia burgdorferi. PLOS ONE 7:e38171. doi: 10.1371/journal.pone.0038171

Wilks, J. C., Kitko, R. D., Cleeton, S. H., Lee, G. E., Ugwu, C. S., Jones, B. D., et al. (2009). Acid and base stress and transcriptomic responses in Bacillus subtilis. Appl. Environ. Microbiol. 75, 981-990. doi: 10.1128/AEM.01652-08

Wilks, J. C., and Slonczewski, J. L. (2007). pH of the cytoplasm and periplasm of Escherichia coli: rapid measurement by green fluorescent protein fluorimetry. J. Bacteriol. 189, 5601-5607. doi: 10.1128/JB.00615-07

Xu, H., Caimano, M. J., Lin, T., He, M., Radolf, J. D., Norris, S. J., et al. (2010). Role of acetyl-phosphate in activation of the Rrp2-RpoN-RpoS pathway in Borrelia burgdorferi. PLOS Pathog. 6:e1001104. doi: 10.1371/journal.ppat.1001104

Yang, X., Goldberg, M. S., Popova, T. G., Schoeler, G. B., Wikel, S. K., Hagman, K. E., et al. (2000). Interdependence of environmental factors influencing reciprocal patterns of gene expression in virulent Borrelia burgdorferi. Mol. Microbiol. 37, 1470-1479. doi: 10.1046/j.1365-2958.2000.02104.x

Yang, X. F., Alani, S. M., and Norgard, M. V. (2003). The response regulator Rrp2 is essential for the expression of major membrane lipoproteins in Borrelia burgdorferi. Proc. Natl. Acad. Sci. U.S.A. 100, 11001-11006. doi: 10.1073/pnas. 1834315100

Conflict of Interest Statement: The authors declare that the research was conducted in the absence of any commercial or financial relationships that could be construed as a potential conflict of interest.

Copyright (C) 2017 Dulebohn, Richards, Su, Lawrence and Gherardini. This is an open-access article distributed under the terms of the Creative Commons Attribution License (CC BY). The use, distribution or reproduction in other forums is permitted, provided the original author(s) or licensor are credited and that the original publication in this journal is cited, in accordance with accepted academic practice. No use, distribution or reproduction is permitted which does not comply with these terms. 